Supporting Materials:

\title{
Trapped intermediates in crystals of the FMN-dependent oxidase PhzG provide insight into the final steps of phenazine biosynthesis
}

\author{
Ningna $\mathrm{Xu}^{\mathrm{ab}}$, Ekta Gahanji Ahuja ${ }^{\mathrm{bc}}$, Petra Janning ${ }^{\mathrm{d}}$, Dmitri Valeryevich Mavrodi ${ }^{\mathrm{e}}$, Linda S.
} Thomashow $^{\dagger}$ and Wulf Blankenfeldt ${ }^{\text {abg* }}$

aLehrstuhl für Biochemie, Universität Bayreuth, Universitätsstraße 30, Bayreuth, 95447, Germany, ${ }^{b}$ Physikalische Biochemie, Max-Planck-Institut für Molekulare Physiologie, Otto-Hahn-Straße 11, Dortmund, 44227, Germany, 'Mithros Chemicals Pvt. Ltd., IKP Knowledgepark, Turkapally, Hyderabad, Andhra Pradesh, 500078, India, ${ }^{d}$ Chemische Biologie, Max-Planck-Institut für Molekulare Physiologie, Otto-Hahn-Straße 11, Dortmund, 44227, Germany, ${ }^{e}$ Department of Plant Pathology, Washington State University, Pullman, Washington, WA 99164-6430, United States, ${ }^{f}$ Root Disease and Biological Control Research Unit, Agricultural Research Service, USDA-ARS, Pullman, Washington, WA 99164-4234, United States, and ${ }^{9}$ Bayreuther Zentrum für Molekulare Biowissenschaften (BZMB), Universität Bayreuth, Germany

Correspondence email: wulf.blankenfeldt@uni-bayreuth.de 


\section{Figure S1}
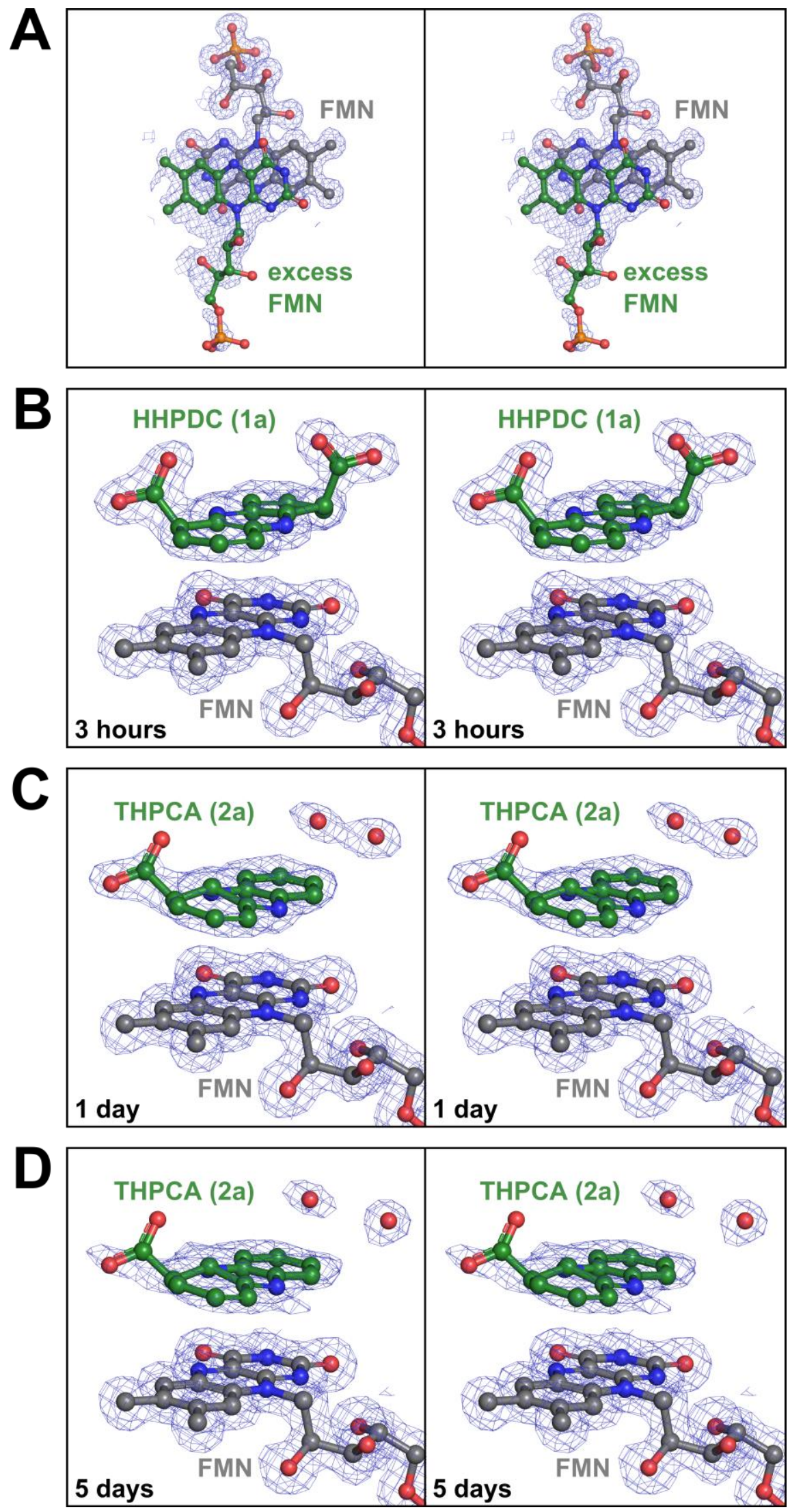

Stereo plots of $1 \sigma|2 \mathrm{FO}-\mathrm{FC}|$ electron density maps of the cofactor FMN and bound ligands in crystals of PhzG from Pseudomonas fluorescens 2-79 at the end of the refinement. 


\section{Figure S2}
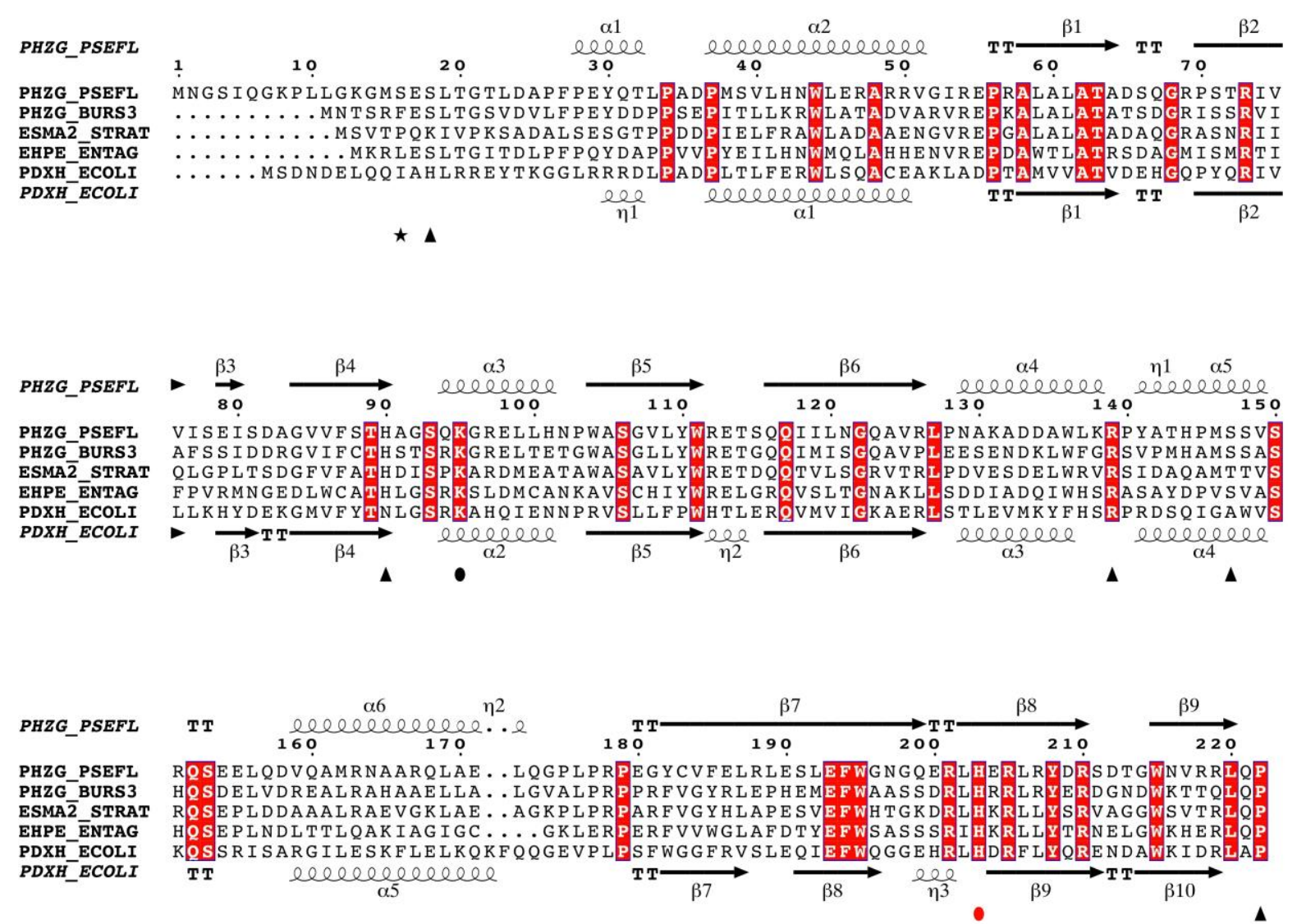

Structure-based sequence alignment of PhzGs from Pseudomonas fluorescens 2-79 (PHZG_PSEFL, Uniprot entry Q51793), Burkholderia lata 383 (PHZG_BURS3, Uniprot entry Q396C5), Streptomyces antibioticus Tü 2706 (ESMA2_STRAT; Uniprot entry H6ACX8_STRAT) and Enterobacter agglomerans Eh1087 (EHPE_ENTAG, Uniprot entry Q8GPH1_ENTAG) with pyridoxine 5'-phosphate oxidase PdxH from E. coli (PDXH_ECOLI, Uniprot entry P0AFI7). Secondary structure elements of $P$. fluorescens 2-79 PhzG (PDB entry 1TY9, (Parsons et al., 2004)) and E. coli PdxH (PDB entry 1G79, (Safo et al., 2001)) are shown on top and at the bottom of each block.

A star marks the first $\mathrm{N}$-terminal residue visible in the electron density of $P$. fluorescens 2-79 $\mathrm{PhzG}$ in complex with hexahydro-phenazine-1,6-dicarboxylic acid (HHPDC, see main text). Black triangles indicate residues involved in the binding of HHPDC, red and black spheres mark residues postulated to be directly or indirectly involved in the enzymatic mechanism of PhzG, respectively (see Fig. 5C of the main text). The sequences were aligned with PROMALS3D (Pei et al., 2008) and rendered with ESPript (Gouet et al., 2003). 


\section{Figure S3}

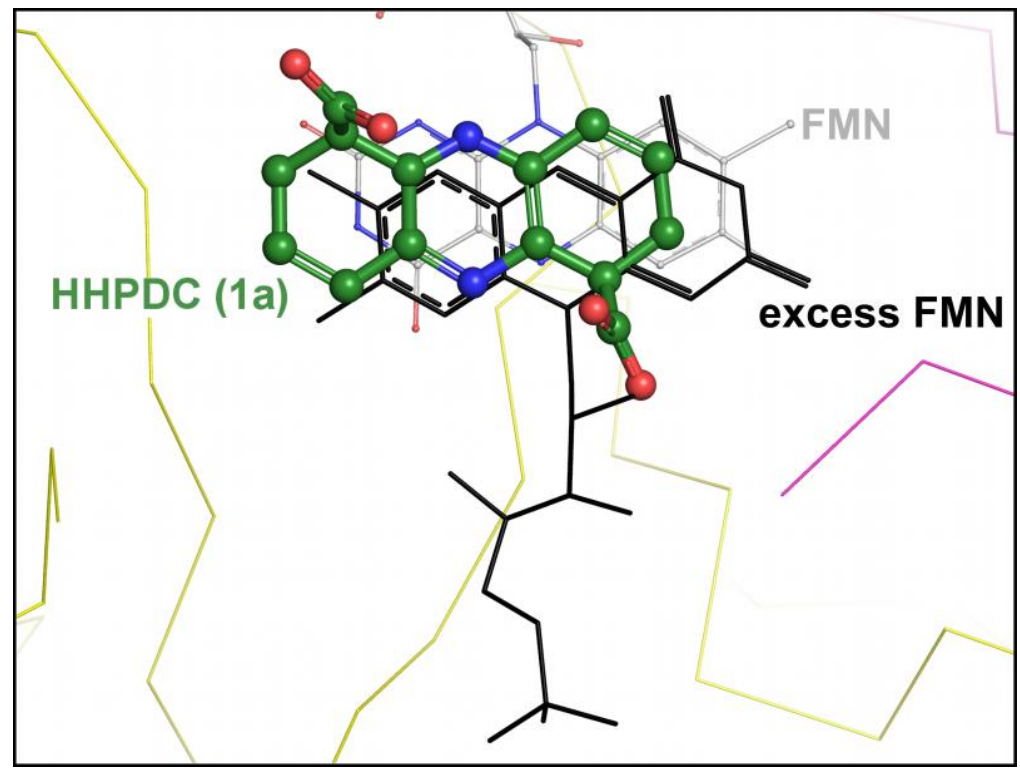

Superimposition of $P$. fluorescens 2-79 PhzG complexes with HHPDC (green) and excess FMN (thin black lines).

\section{Figure S4}

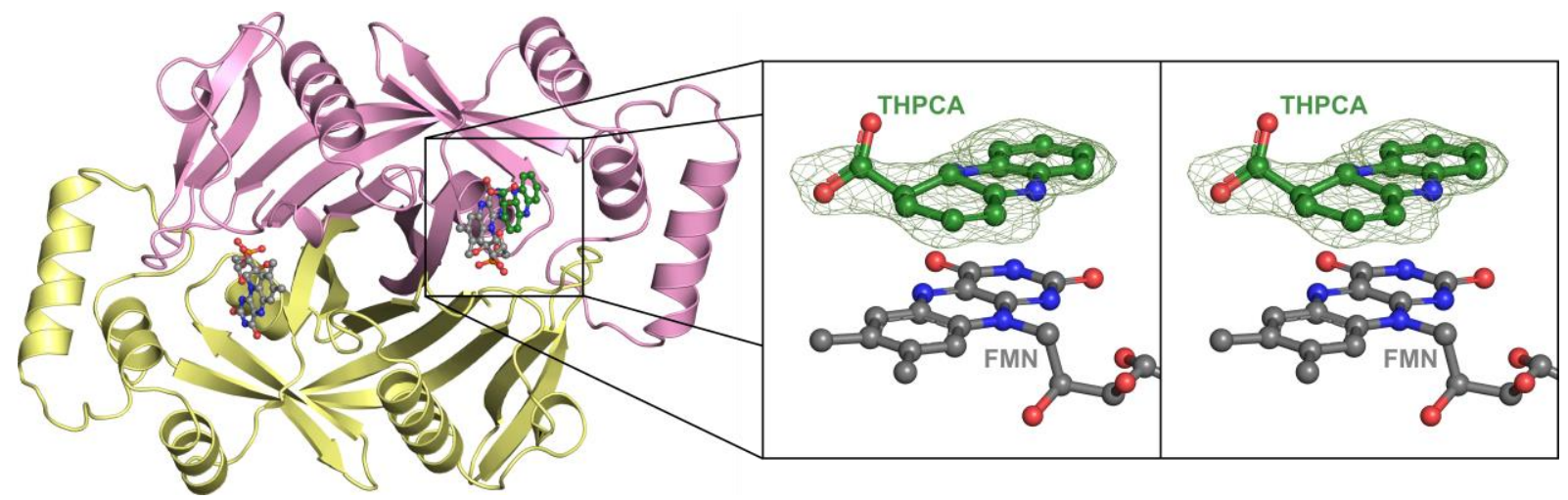

Crystal structure of PhzG from Burkholderia lata 383 in complex with tetrahydro-phenazine1-carboxylic acid (THPDC). The magnified insert shows a stereo plot of $3 \sigma|\mathrm{FO}-\mathrm{FC}|$ difference electron density of the ligand before incorporation into the structural model. 
Table S1 Data collection statistics for Burkholderia lata $383 \mathrm{PhzG}$

Values in parentheses are for the highest resolution shell. Both data sets were collected from single crystals on beamline X10SA of the Swiss Light Source (Paul Scherrer Institute, Villigen, Switzerland).

\begin{tabular}{lll}
\hline Dataset & Apo-structure & complex with intermediate 2a \\
Wavelength $(\AA)$ & 0.99988 & 0.97886 \\
Resolution range $(\AA)$ & $47-1.53(1.56-1.53)$ & $47-1.59(1.62-1.59)$ \\
Space group & $\mathrm{P} 3_{1}$ & $\mathrm{P} 3_{1}$ \\
Unit cell parameters $(\AA)$ & $94.42,94.42,51.75$ & $93.65,93.65,51.41$ \\
Mosaicity $\left(^{\circ}\right)^{\dagger}$ & 0.323 & 0.169 \\
Total No. of measured reflections & $339919(14939)$ & $385105(14994)$ \\
Unique reflections & $77682(3888)$ & $67792(3389)$ \\
Multiplicity & $4.4(3.8)$ & $5.7(4.4)$ \\
Mean $\mathrm{I} / \sigma(\mathrm{I})_{\text {Completeness }(\%)}^{10.1(2.0)}$ & $16.0(2.1)$ \\
$\mathrm{R}_{\text {meas }}(\%)^{\ddagger}$ & $99.7(99.9)$ & $99.9(100)$ \\
$\mathrm{R}_{\text {pim }}(\%)^{\$}$ & $8.7(87.4)$ & $6.3(80.5)$ \\
\hline
\end{tabular}

Mosaicity values reported by XDS (Kabsch, 2010)

${ }^{\ddagger} \mathrm{R}_{\text {meas }}=\Sigma_{\mathrm{hkl}}(\mathrm{N} /(\mathrm{N}-1))^{1 / 2} \Sigma_{\mathrm{i}}\left|\mathrm{I}_{\mathrm{i}}(\mathrm{hkl})-<\mathrm{I}(\mathrm{hkl})>\right| / \Sigma_{\mathrm{hkl}} \Sigma_{\mathrm{i}} \mathrm{I}_{\mathrm{i}}(\mathrm{hkl})$, where $\mathrm{N}$ is the number of observations of the reflection with index hkl and $\mathrm{I}_{\mathrm{i}}$ is the intensity of its $i^{\text {th }}$ observation.

${ }^{\$} \mathrm{R}_{\mathrm{pim}}=\Sigma_{\mathrm{hkl}}(1 /(\mathrm{N}-1))^{1 / 2} \Sigma_{\mathrm{i}}\left|\mathrm{I}_{\mathrm{i}}(\mathrm{hkl})-<\mathrm{I}(\mathrm{hkl})>\right| / \Sigma_{\mathrm{hkl}} \Sigma_{\mathrm{i}} \mathrm{I}_{\mathrm{i}}(\mathrm{hkl})($ Weiss, 2001). 
Table S2 Refinement statistics for Burkholderia lata $383 \mathrm{PhzG}$

Values in parentheses are for the highest resolution shell.

\begin{tabular}{|c|c|c|}
\hline Dataset & Apo-structure & complex with intermediate $\mathbf{2 a}$ \\
\hline Resolution range ( $\AA$ ) & $47-1.53(1.55-1.53)$ & $47-1.59(1.61-1.59)$ \\
\hline $\mathrm{R}_{\text {cryst }}(\%)$ & $13.3(26.0)$ & $11.3(20.7)$ \\
\hline $\mathrm{R}_{\text {free }}(\%)$ & $17.9(30.6)$ & $15.8(27.0)$ \\
\hline \multicolumn{3}{|l|}{ No. of non-H atoms } \\
\hline Protein & 3621 & 3569 \\
\hline Ion & - & - \\
\hline Ligand & 68 & 85 \\
\hline Water & 544 & 490 \\
\hline \multicolumn{3}{|l|}{ R.m.s. deviations } \\
\hline Bonds $(\AA)$ & 0.012 & 0.019 \\
\hline Angles $\left({ }^{\circ}\right)$ & 1.360 & 1.702 \\
\hline \multicolumn{3}{|l|}{ Average B factors $\left(\AA^{2}\right)$} \\
\hline Protein & 27 & 25 \\
\hline Ion & - & - \\
\hline Ligand & 16 & 20 \\
\hline Water & 39 & 39 \\
\hline \multicolumn{3}{|l|}{ Ramachandran plot } \\
\hline Favoured regions (\%) & 98.3 & 98.0 \\
\hline Outliers (\%) & 0 & 0 \\
\hline MolProbity score ${ }^{\#}$ & 1.31 & 1.34 \\
\hline PDB entry code & 4HMW & $4 \mathrm{HMX}$ \\
\hline
\end{tabular}

\#As reported by MolProbity at http://molprobity.biochem.duke.edu/ (Chen et al., 2010) 


\section{Supporting references}

Chen, V. B., Arendall, W. B., Headd, J. J., Keedy, D. A., Immormino, R. M., Kapral, G. J., Murray, L. W., Richardson, J. S., \& Richardson, D. C. (2010). Acta Crystallogr. D Biol. Crystallogr. 66, 12-21.

Gouet, P., Robert, X., \& Courcelle, E. (2003). Nucleic Acids Research. 31, 3320-3323.

Kabsch, W. (2010). Acta Crystallogr. D Biol. Crystallogr. 66, 125-132.

Parsons, J. F., Calabrese, K., Eisenstein, E., \& Ladner, J. E. (2004). Acta Crystallogr. D Biol. Crystallogr. 60, 2110-2113.

Pei, J., Kim, B.-H., \& Grishin, N. V. (2008). Nucleic Acids Res. 36, 2295-2300.

Safo, M. K., Musayev, F. N., Di Salvo, M. L., \& Schirch, V. (2001). J. Mol. Biol. 310, 817826.

Weiss, M. S. (2001). Journal of Applied Crystallography. 34, 130-135. 